\title{
Conhecimento do enfermeiro da atenção primária à saúde sobre a indicação de coberturas especiais
}

\section{Knowledge of nurses in primary health care about the indication of special coverage \\ Conocimiento del enfermero de la atención primaria a la salud sobre la indicación de coberturas especiales}

\author{
Cristiano Caveião ${ }^{1,2}$, Ana Paula Hey³, Willian Barbosa Sales², Edson Luís Pereira Tavares², \\ Elisangela de Souza², Marly Marton Bittencourt Gervásio da Silva ${ }^{4}$
}

ORCID IDS

Caveião C (D) https://orcid.org/0000-0003-2664-7542

Sales WB (iD https://orcid.org/0000-0003-2367-8271

Tavares ELP (D) https://orcid.org/0000-0002-5833-8763

Souza E (D) https://orcid.org/ 0000-0001-7194-9764

Silva MMBG (D) https://orcid.org//0000-0002-0940-8382

\section{COMO CITAR}

Caveião C, Hey AP, Sales WB, Tavares ELP, de Souza E, da Silva MMBG. Conhecimento do enfermeiro da atenção primária à saúde sobre a indicação de coberturas especiais. ESTIMA, Braz. J. Enterostomal Ther., 16: e3118. https://doi.org/10.30886/estima.v16.562 PT.

\section{RESUMO}

Objetivo: Identificar o conhecimento dos enfermeiros da atenção primária à saúde de um município sobre indicação de coberturas especiais. Método: Estudo exploratório, descritivo, com abordagem quantitativa realizado com 101 enfermeiros em 109 unidades de saúde da atenção primária em saúde por meio de instrumento estruturado. Os dados foram analisados estatisticamente com a distribuição de frequência, percentis, média, mediana, desvio padrão, coeficiente de variação, intervalo de confiança, p-valor e teste de igualdade de duas proporções para comparar o grupo que tem capacitação/aperfeiçoamento na área com o grupo que não tem. Resultados: Sobre a indicação da cobertura, 79,2\% referiu que se destina a controlar o exsudato e o tecido não viável, e a indicação com maior porcentagem de acertos para utilização em feridas foi a cobertura hidrofibra de prata e hidrocoloide com prata. Conclusão: Enfermeiros da atenção primária com cursos de capacitação/aperfeiçoamento não têm conhecimento suficiente para indicação das coberturas.

DESCRITORES: Enfermagem; Curativos oclusivos; Conhecimento; Atenção primária à saúde; Estomaterapia.

\footnotetext{
${ }^{1}$ Centro Universitário Internacional - Escola Superior de Saúde, Biociências, Meio Ambiente e Humanidades - Curitiba/PR - Brasil. ${ }^{2}$ Centro Universitário Autônomo do Brasil - Escola de Ciências da Saúde - Curso de Enfermagem - Curitiba/PR - Brasil. ${ }^{3}$ Universidade Tuiuti do Paraná - Faculdade de Ciências Biológicas e da Saúde - Curso de Enfermagem - Curitiba/PR - Brasil. ${ }^{4}$ Universidade Positivo - Escola de Ciências da Saúde - Curso de Enfermagem - Curitiba/PR - Brasil. Autor correspondente: Cristiano Caveião | Rua Treze de Maio, 538 - Centro | CEP: 80510-030 - Curitiba/PR | E-mail: cristiano_caveiao@hotmail.com Recebido: Dez. 11, 2017 | Aceito: Nov. 21, 2018
} 


\section{ABSTRACT}

Objective: To identify the knowledge of nurses in primary health care in a municipality about the indication of special coverage. Method: An exploratory, descriptive study with a quantitative approach performed with 101 nurses in 109 health units of primary health care through a structured instrument. The data were statistically analyzed with frequency distribution, percentiles, average, median, standard deviation, coefficient of variation, confidence interval, p-value and two-proportion equality test to compare the group that has qualification/improvement in the area with the group do not have. Results: About the indication of the coverage, $79.2 \%$ reported that it is intended to control exudate and non-viable tissue, and the indication with the highest percentage of correct answers for use in wounds was the silver hydrofibra coverage and hydrocolloid with silver. Conclusion: Primary care nurses with qualification/ improvement courses do not have enough knowledge to indicate the coverage.

DESCRIPTORS: Nursing; Occlusive healings; Knowledge; Primary health care; Stomatherapy.

\section{RESUMEN}

Objetivo: Identificar el conocimiento de los enfermeros de la atención primaria a la salud de un municipio sobre la indicación de coberturas especiales. Método: Estudio exploratorio, descriptivo, con abordaje cuantitativo realizado con 101 enfermeros en 109 unidades de salud de la atención primaria en salud por medio de instrumento estructurado. Los datos fueron analizados estadísticamente con la distribución de frecuencia, percentiles, promedio, mediana, desvío estándar, coeficiente de variación, intervalo de confianza, p-valor y prueba de igualdad de dos proporciones para comparar el grupo que tiene capacitación/perfeccionamiento en el área con el grupo que no lo tiene. Resultados: Sobre la indicación de la cobertura, 79,2 \% indicó que está destinado a controlar el exudado y el tejido no viable, y la indicación con mayor porcentaje de aciertos para utilización en heridas fue la cobertura hidrofibra de plata e hidrocoloide con plata. Conclusión: Enfermeros de la atención primaria con cursos de capacitación/perfeccionamiento no tienen conocimiento suficiente para la indicación de las coberturas.

DESCRIPTORES: Enfermería; Curativos oclusivos; Conocimiento; Atención primaria a la salud; Estomaterapia..

\section{INTRODUÇÃO}

A atenção primária à saúde (APS) é um conjunto de estratégias de organização para atendimento de forma regionalizada, contínua e sistematizada no qual se integram ações preventivas, curativas, de responsabilização, de humanização, da equidade, da participação individual, social e da comunidade ${ }^{1}$. Segundo o Ministério da Saúde, a APS é um serviço especializado e porta de entrada preferencial para o Sistema Único de Saúde (SUS), que ocorre de forma integral e universal, envolvendo ações de promoção, proteção e recuperação da saúde ${ }^{2}$. Na rede de serviços da Secretaria Municipal da Saúde (SMS) do município pesquisado, visando a melhor assistência na APS, o serviço passou a oferecer, em 2015, tratamento de feridas agudas e crônicas de diferentes etiologias.

$\mathrm{Na}$ APS, faz parte da rotina diária do enfermeiro o tratamento de feridas ${ }^{3}$. Com a avaliação do paciente de forma sistematizada, contempla a anamnese, o exame físico, o diagnóstico de enfermagem, as intervenções, as prescrições de enfermagem e os encaminhamentos. Durante a consulta, desenvolve-se o plano de cuidados para a escolha da cobertura ideal e o acompanhamento da evolução da ferida, com intuito de observar a eficácia do tratamento. Essas medidas podem reduzir as complicações das feridas, como infecções e amputações ${ }^{4}$.

O conhecimento do enfermeiro e suas competências em cuidados de feridas na APS são regulamentados pela Resolução No 567, de 29 de janeiro de 2018, a qual define as atribuições do enfermeiro no cuidado com feridas: realizar curativos, supervisionar a equipe de enfermagem nos cuidados com feridas, abertura de consultórios para cuidados com feridas, prescrever medicamentos e coberturas para feridas e executar debridamento químico e mecânico em feridas 5 .

A ferida é considerada a perda da continuidade do tegumento, representada não apenas pela ruptura da pele e do tecido celular subcutâneo, mas também, em alguns casos, músculos, tendões e ossos. Pode ser classificada quanto à etiologia, complexidade e tempo de existência ${ }^{6}$. Os fatores predisponentes mais comuns das feridas são hospitalização prolongada, idade, obesidade, estado nutricional e diabetes melito 7 . Seu tratamento depende da evolução do processo de reparação tecidual, incluindo métodos clínicos e cirúrgicos, sendo o curativo e a cobertura especial o método clínico 
mais frequentemente utilizado ${ }^{8}$. Para que apresente êxito, requer avaliação realizada pelo enfermeiro para que possa indicar qual cobertura especial é adequada para cada objetivo.

O curativo é o meio terapêutico que consiste na limpeza, com aplicação de procedimentos assépticos, que vai desde a irrigação com solução fisiológica até as coberturas especiais que poderão auxiliar em cada momento do processo cicatricial. Essas coberturas foram desenvolvidas com objetivos diferentes para cada situação, porém bem definidos quanto à sua indicação'.

A escolha da cobertura especial e do material mais adequado para o tratamento inclui o conhecimento fisiopatológico e bioquímico dos mecanismos de cicatrização e reparação tissular, etiologia, localização da lesão, tamanho da ferida, condições clínicas e fases do processo de cicatrização. As coberturas podem ser classificadas como passivas, com função apenas de cobrir as feridas (gaze; gaze não aderente) e bioativas (liberam elementos necessários à cicatrização e aceleram a cicatrização $)^{4,5,9,10}$. Entretanto, a escolha da cobertura ideal merece atenção especial do enfermeiro, baseando-se em seu conhecimento da avaliação das feridas. Ainda requer levar em consideração as coberturas disponíveis no mercado, bem como sua composição, mecanismos de ação, indicação, utilização e o intervalo entre as trocas.

Portanto, diante dessa contextualização, a indicação da cobertura adequada para cada tipo de ferida pode contribuir para o processo cicatricial, evitando complicações tardias, tais como sepses de partes moles e até amputação. Além da melhora do quadro do paciente, a prescrição e a aplicação correta das coberturas auxiliam no tratamento rápido e eficaz. Este estudo teve como objetivo identificar o conhecimento dos enfermeiros da APS sobre indicação de coberturas especiais.

\section{MÉTODOS}

Trata-se de estudo exploratório, descritivo, com abordagem quantitativa realizado com enfermeiros em 109 unidades de saúde da APS de um município da Região Sul do Brasil. Foram convidados para participar do estudo 321 enfermeiros que atuam na APS, no entanto 220 recusaram participar ou estavam ausentes durante o período do estudo. A amostra foi composta por 101 enfermeiros.

Os critérios de inclusão foram enfermeiros lotados na APS e que realizam prescrição de coberturas especiais e os critérios de exclusão foram enfermeiros ausentes no período de coleta de dados, em férias ou em licença (tratamento de saúde, maternidade, conselho tutelar, mandato seletivo, tratamento de saúde familiar, sem vencimentos, prêmio decênio e prêmio quinquênio).

Após confirmação com a autoridade sanitária local (ASL) das unidades de saúde para levantamento do número de enfermeiros em cada unidade, foram enviados convites a todos e entregues o instrumento de coleta das informações e o Termo de Consentimento Livre e Esclarecido. A entrevista foi agendada em data e horário predefinidos com a ASL e ocorreu na própria unidade de saúde, em local privativo.

A coleta das informações ocorreu em setembro de 2017, por meio de instrumento estruturado, elaborado pelos próprios pesquisadores, contendo questões fechadas de múltipla escolha relacionadas à indicação das coberturas especiais disponibilizadas pela SMS e utilizadas pelos enfermeiros. A relação das coberturas especiais foi obtida por meio do protocolo de tratamento de feridas, sendo as seguintes coberturas: ácidos graxos essenciais; alginato de cálcio e sódio; carvão ativado; hidrogel; espuma a base de poliuretano com prata; gaze não aderente; hidrofibra com prata; e placa de hidrocoloide. Podem ocorrer variações nas coberturas especiais devido às atualizações no protocolo que ainda não foram divulgadas. Após a construção do instrumento, esse passou pela avaliação de aparência e de conteúdo por duas enfermeiras estomaterapeutas.

Após a coleta dos dados, esses foram digitados em planilha do Microsoft Excel Office ${ }^{\circledR}$ 2010, utilizando a dupla digitação para não ocorrer margem de erros. Para análise estatística dos dados, utilizou-se o software Statistical Package for the Social Science (SPSS) versão 17.0 e os resultados foram apresentados por meio de distribuição de frequência, percentis, média, mediana, desvio padrão (DP), coeficiente de variação, intervalo de confiança, $\mathrm{p}$-valor e teste de igualdade de duas proporções para comparar o grupo que tem capacitação/aperfeiçoamento na área com o grupo que não tem. Os resultados foram analisados por meio da literatura sobre o tema. O erro amostral calculado foi de $5 \%$.

Os aspectos éticos foram respeitados em todas as etapas do estudo, de acordo com a Resolução No 466, de 12 de dezembro de 2012, que trata das recomendações que regulamentam a pesquisa envolvendo seres humanos ${ }^{11}$. O estudo foi aprovado pelo comitê de ética em pesquisa envolvendo seres humanos (CAEE 69191517.3.0000.0101, parecer 2.230.211). 


\section{RESULTADOS}

O estudo permitiu analisar o conhecimento dos enfermeiros das unidades da APS do município sobre a indicação de coberturas especiais para tratamento de feridas. Os dados obtidos possibilitaram a caracterização dos enfermeiros quanto aos aspectos sociodemográficos.

Participaram do estudo 101 enfermeiros, sendo 96\% (97) do gênero feminino, com média de idade de 44,2 anos (DP $\pm 8,4$ ), mediana de 43 anos, mínimo de 22 e máximo de 64 anos. Quanto ao ano de conclusão da graduação em enfermagem, a maioria dos enfermeiros finalizou seus cursos nas décadas de $2000(56,4 \%, \mathrm{n}=57)$ e na de $1990(21,8 \%$, $\mathrm{n}=22)$. Referente à especialização, $84,2 \%(\mathrm{n}=85)$ têm curso de especialização lato sensu (Tabela 1), sendo que $45,8 \%$ ( $n=38)$ na área de saúde da família/saúde coletiva e 3\% ( $n=3)$ são mestres. Durante a formação, $65,3 \%(n=66)$ relataram ter estudado conteúdos relacionados a coberturas especiais.

Tabela 1. Caracterização dos enfermeiros quanto à década de formação e especialização latu sensu, 2017.

\begin{tabular}{|c|c|c|}
\hline Variável & n (\%) & p-valor* \\
\hline \multicolumn{3}{|l|}{ Conclusão do curso (década) } \\
\hline 1970 & $2(2,0)$ & $<0,001$ \\
\hline 1980 & $9(8,9)$ & $<0,001$ \\
\hline 1990 & $22(21,8)$ & $<0,001$ \\
\hline 2000 & $57(56,4)$ & Referência \\
\hline 2010 & $11(10,9)$ & $<0,001$ \\
\hline \multicolumn{3}{|l|}{ Pós-graduação } \\
\hline Administração & $2(2,4)$ & $<0,001$ \\
\hline Auditoria & $6(7,2)$ & $<0,001$ \\
\hline Emergência & $12(14,5)$ & $<0,001$ \\
\hline Enfermagem do trabalho & $6(7,2)$ & $<0,001$ \\
\hline $\begin{array}{l}\text { Enfermagem em centro } \\
\text { cirúrgico }\end{array}$ & $1(1,2)$ & $<0,001$ \\
\hline Estomaterapia & $2(2,4)$ & $<0,001$ \\
\hline Gestão em saúde & $5(6,0)$ & $<0,001$ \\
\hline Gestão pública & $2(2,4)$ & $<0,001$ \\
\hline Infecção hospitalar/epidemiologia & $2(2,4)$ & $<0,001$ \\
\hline Obstetrícia & $6(7,2)$ & $<0,001$ \\
\hline Pediatria & $1(1,2)$ & $<0,001$ \\
\hline Saúde da família/saúde coletiva & $38(45,8)$ & $<0,001$ \\
\hline
\end{tabular}

*Teste de igualdade de duas proporções.

Com relação ao tempo de atuação na APS, a média foi de 13,1 anos (DP $\pm 6,9$ ), com mediana de 11 , mínimo de 1 e máximo de 32 anos. Quando perguntados sobre a quantidade de coberturas especiais realizada por mês no serviço, a média foi de 8,1 ( $\mathrm{DP} \pm 12,2)$, com mediana de 4. Referente à realização de cursos de capacitação em tratamento de feridas, $77,2 \%(n=78)$ realizaram e $93,1 \%$ ( $n=94)$ fizeram cursos de aperfeiçoamento em 2017.

Quando perguntados sobre a cobertura ideal, 79,2\% $(\mathrm{n}=80)$ responderam que deve controlar exsudato e tecido não viável e ser biocompatível (Tabela 2).

Tabela 2. Caracterização dos enfermeiros quanto à indicação da cobertura, 2017.

\begin{tabular}{lcc}
\multicolumn{1}{c}{ A cobertura ideal deve } & $\mathbf{n}(\%)$ & p-valor* \\
\hline $\begin{array}{l}\text { Controlar exsudato e tecido } \\
\text { não viável }\end{array}$ & $80(79,2)$ & Referência \\
\hline $\begin{array}{l}\text { Manter o leito da ferida úmido } \\
\text { e a pele circundante seca }\end{array}$ & $70(69,3)$ & 0,108 \\
\hline $\begin{array}{l}\text { Ter permeabilidade seletiva } \\
\text { para entrada de oxigênio }\end{array}$ & $57(56,4)$ & $<0,001$ \\
\hline Promover isolamento térmico & $44(43,6)$ & $<0,001$ \\
\hline Ser biocompatível & $80(79,2)$ & Referência \\
\hline
\end{tabular}

*Teste de igualdade de duas proporções

Com relação à indicação de cada cobertura especial utilizada na APS e que constam do Protocolo de Tratamento de Feridas da SMS, destaca-se que foram listadas as coberturas especiais e a indicação de cada uma delas, cabendo ao enfermeiro escolher qual a melhor indicação para cada cobertura. Também foi realizada a comparação entre o grupo de enfermeiros que tem capacitação/aperfeiçoamento em feridas e o grupo que não tem e observa-se que não existe diferença estatisticamente significante entre os grupos para a distribuição dos tratamentos, ou seja, os grupos são homogêneos/iguais (Tabela 3).

\section{DISCUSSÃO}

A presença feminina é destaque na enfermagem desde a práticas antigas, devido à arte do cuidar das mais diferentes formas, que era concebida por meio de saberes passados de geração para geração. Sob influência de Florence Nightingale, ocorreu a feminização da profissão devido à ideia da vocação das mulheres para o cuidar ${ }^{11}$.

Com relação à formação, houve maior predominância na última década, devido à grande expansão da oferta 
Tabela 3. Conhecimento dos enfermeiros para a indicação da cobertura especial, 2017.

\begin{tabular}{|c|c|c|c|c|c|}
\hline \multirow{2}{*}{ Cobertura } & \multicolumn{2}{|c|}{ Grupo geral } & \multirow{2}{*}{$\begin{array}{c}\text { Grupo com } \\
\text { capacitação/ } \\
\text { aperfeiçoamento } \\
n(\%)\end{array}$} & \multirow{2}{*}{$\begin{array}{c}\text { Grupo sem } \\
\text { capacitação/ } \\
\text { aperfeiçoamento } \\
\text { n (\%) }\end{array}$} & \multirow{2}{*}{ p-valor* } \\
\hline & n (\%) & p-valor* & & & \\
\hline \multicolumn{6}{|l|}{ Alginato de cálcio } \\
\hline Autolítico & $43(42,6)$ & $<0,001$ & $34(24,8)$ & $9(25,7)$ & 0,913 \\
\hline Controle de umidade & $67(66,3)$ & Referência & $56(40,9)$ & $11(31,4)$ & 0,306 \\
\hline \multicolumn{6}{|l|}{ Espuma de prata } \\
\hline Antimicrobiano & $84(83,2)$ & Referência & $66(30,6)$ & $18(30,5)$ & 0,994 \\
\hline Autolítico & $33(32,7)$ & $<0,001$ & $27(12,5)$ & $6(10,2)$ & 0,625 \\
\hline Controle de odor & $58(57,4)$ & $<0,001$ & $43(19,9)$ & $15(25,4)$ & 0,357 \\
\hline \multicolumn{6}{|l|}{ Gaze não aderente } \\
\hline Manutenção umidade & $70(69,3)$ & Referência & $59(49,2)$ & $11(45,8)$ & 0,766 \\
\hline \multicolumn{6}{|l|}{ Hidrocoloide } \\
\hline Autolítico & $42(41,6)$ & $<0,001$ & $36(21,4)$ & $6(15,4)$ & 0,398 \\
\hline Hidratação & $42(41,6)$ & $<0,001$ & $34(20,2)$ & $8(20,5)$ & 0,969 \\
\hline Manutenção umidade & $70(69,3)$ & Referência & $58(34,5)$ & $12(30,8)$ & 0,655 \\
\hline \multicolumn{6}{|l|}{ Hidrocoloide com prata } \\
\hline Antimicrobiano & $85(84,2)$ & Referência & $66(31,6)$ & $19(34,5)$ & 0,675 \\
\hline Autolítico & $34(33,7)$ & $<0,001$ & $28(13,4)$ & $6(10,9)$ & 0,624 \\
\hline Controle de odor & $43(42,6)$ & $<0,001$ & $33(15,8)$ & $10(18,2)$ & 0,669 \\
\hline Controle de umidade & $34(33,7)$ & $<0,001$ & $23(11,0)$ & $5(9,1)$ & 0,682 \\
\hline Hidratação & $30(29,7)$ & $<0,001$ & $24(11,5)$ & $6(10,9)$ & 0,905 \\
\hline Manutenção umidade & $44(43,6)$ & $<0,001$ & $35(16,7)$ & $9(16,4)$ & 0,946 \\
\hline \multicolumn{6}{|l|}{ Hidrofibra de prata } \\
\hline Antimicrobiano & $89(88,1)$ & Referência & $71(29,2)$ & $18(34,0)$ & 0,495 \\
\hline Autolítico & $39(38,6)$ & $<0,001$ & $32(13,2)$ & $7(13,2)$ & 0,994 \\
\hline Controle de odor & $53(52,5)$ & $<0,001$ & $43(17,7)$ & $10(18,9)$ & 0,840 \\
\hline Controle de umidade & $66(65,3)$ & $<0,001$ & $53(21,8)$ & $13(24,5)$ & 0,667 \\
\hline Manutenção umidade & $38(37,6)$ & $<0,001$ & $34(14,0)$ & $4(7,5)$ & 0,204 \\
\hline \multicolumn{6}{|l|}{ Hidrogel } \\
\hline Autolítico & $81(80,2)$ & Referência & $65(39,6)$ & $16(32,7)$ & 0,377 \\
\hline Hidratação & $58(57,4)$ & $<0,001$ & $43(26,2)$ & $15(30,6)$ & 0,544 \\
\hline Manutenção umidade & $57(56,4)$ & $<0,001$ & $42(25,6)$ & $15(30,6)$ & 0,488 \\
\hline
\end{tabular}

*Teste de igualdade de duas proporções.

de cursos de graduação em enfermagem ${ }^{12}$, oriunda dos incentivos do governo, como bolsas de estudos.

Após a implantação das Diretrizes Curriculares Nacional de Enfermagem ${ }^{13}$, em 2001, não ocorreu a discriminação de conteúdo específicos para a formação do enfermeiro. Salienta-se que os conteúdos referentes à avaliação e à indicação de coberturas especiais são tópicos à parte para cursos de capacitação, atualização e especialização.
Os profissionais da área de enfermagem cada vez mais investem no aperfeiçoamento dos seus conhecimentos por meio de cursos de pós-graduação e capacitação, para suprirem as necessidades do mercado, que se torna cada dia mais competitivo $^{14,15}$. Devido à principal atuação do enfermeiro na saúde pública, esses especializam-se nessa área.

A cobertura ideal deve garantir um ambiente adequado para a cicatrização, controlando a umidade, permitindo o controle do excesso de exsudato, permitindo a troca 
gasosa, proporcionando isolamento térmico, controlando a proliferação de bactérias, impedindo o contato com partículas e tóxicos contaminadores da ferida e garantindo a possibilidade de remoção sem trauma ${ }^{16,17}$. A escolha do tipo de cobertura está diretamente relacionada à sua função que pode ser: proteção e absorção de umidade; absorção de exsudato e odores; desbridamento; limpeza e prevenção da contaminação exógena; compressão para minimizar acúmulo de fluidos; e imobilização ou proteção contra traumatismos mecânicos. A decisão terapêutica deverá ser dinâmica, adaptando-se continuamente à evolução clínica da ferida ${ }^{18,19}$.

O conhecimento do enfermeiro é parte obrigatória e necessária para o exercício de suas atividades, nas quais deve-se relacionar o conhecimento teórico-prático, demonstrando suas capacidades e competências para exercer a profissão. A percepção para o tratamento de feridas abrange tecnologias, indicações, custos e eficácia, dinamizando o tratamento ${ }^{16}$. Ante a isso, é necessário que o profissional tenha conhecimento adequado dos produtos e sobre a indicação das coberturas especiais.

A indicação correta das coberturas faz parte do tratamento de feridas dentre alguns passos que devem ser seguidos: avaliar e reavaliar os pacientes conforme necessidade, pois pode ser necessária a mudança do tipo de cobertura; estabelecer os objetivos referentes à cicatrização; organização do time que envolve o enfermeiro e os cuidadores/familiares para dar sequência ao cuidado e manejo das feridas; estabelecer e implantar um plano, avaliando a necessidade maior da ferida em seu estágio para que se inicie a cicatrização eficaz, ou seja, indicar a cobertura correta para cada etapa da ferida; documentação em prontuários, constando a conduta e a evolução da ferida por meio da avaliação periódica ${ }^{20}$.

Coberturas especiais são dispositivos semioclusivos desenvolvidos para atuar temporariamente como substitutos da pele, promovendo condições ótimas de cicatrização por meio da manutenção de meio úmido ideal ${ }^{17}$.

Com relação aos aspectos relacionados ao conhecimento dos enfermeiros sobre a indicação das coberturas especiais, observou-se que esses não têm especialização na área, ficando abaixo de 50\% em maior parte das indicações de cada cobertura especial.

$\mathrm{Na}$ indicação da cobertura de hidrofibra com prata em outra pesquisa ${ }^{14}$, os enfermeiros também apresentaram menor conhecimento. Para a cobertura de hidrocoloide, nenhum deles soube fazer a indicação para desbridamento autolítico. Para o autor, o resultado da pesquisa justificou-se pelo uso rotineiro da cobertura apenas para prevenção de lesões por pressão ${ }^{16}$.

A cobertura de hidrogel, neste estudo, foi a que obteve maior número de indicações para suas respectivas finalidades. Tal dado assemelha-se à outra pesquisa ${ }^{16}$, que também demonstrou maior quantidade de acertos sobre a indicação dessa cobertura, porém com resultados não satisfatórios. Os dados demonstram que parte dos enfermeiros não detém o devido conhecimento para utilização e prescrição da cobertura. Ainda, pode-se observar, no estudo, que a maioria dos profissionais não cita a função de desbridamento autolítico nas coberturas que têm essa função, como hidrocoloide, hidrocoloide com prata, alginato de cálcio, espuma com prata, hidrofibra e o hidrogel.

Pouco se pode encontrar na literatura sobre o conhecimento específico do enfermeiro referente à indicação de coberturas, o que, se encontrado, demonstra a falta de conhecimento mínimo para a prestação de assistência. Comumente são encontrados protocolos de padronização de coberturas que norteiam o atendimento da assistência à saúde.

Os cuidados devem ser realizados de forma personalizada e de acordo com as necessidades singulares de cada paciente. Às vezes, é necessário enquadrar o paciente em programas específicos, como o de tabagismo, de controle de diabetes e nutricional. A prescrição correta das coberturas é importante, mas o conjunto de cuidados é imprescindível para a eficácia no tratamento ${ }^{20}$.

O serviço de APS tem um protocolo visando à melhoria da qualidade de assistência e padronização das condutas da SMS. Pode-se verificar que as respostas das indicações corretas de cada tipo de cobertura foram semelhantes em todos os grupos. Os dados chamam a atenção para o grupo com especialização/capacitação, que deveria ter um dado assertivo maior em relação ao grupo sem nenhuma capacitação específica.

Os resultados podem ser comparados pela literatura, que identificou que grande parte dos enfermeiros apresentava embasamento sobre a lesão, mas referia dificuldades em identificá-la e também desconhecia seu mecanismo fisiopatológico ${ }^{21}$. A mesma característica também se encontra em outro estudo 6 , no qual poucos entrevistados detalharam corretamente as características da ferida avaliada no atendimento a pessoas com lesão cutânea maligna.

$\mathrm{O}$ enfermeiro que presta atendimento na APS no tratamento de feridas tem como responsabilidade saber a 
indicação correta de cada cobertura e também a aplicabilidade de acordo com a etiologia da ferida e a fase em que ela se encontra, a partir da compreensão sobre a fisiologia da cicatrização ${ }^{18,22}$.

As opções de coberturas acarretam benefícios aos pacientes e maior segurança para os profissionais envolvidos na assistência ${ }^{23}$. Embora os enfermeiros utilizem um manual de padronização de cuidados com feridas, neste estudo as dificuldades de utilizar o que é proposto ocorre devido à falta de produtos recomendados.

O cuidado do enfermeiro com o paciente na APS requer, junto à prática da indicação de coberturas e abordagem, compreender a fisiologia da pele e a cicatrização. Associando o conhecimento científico sobre os tipos de coberturas existentes no mercado, a falta desse conhecimento dificulta o diagnóstico correto do tipo de lesão e também a indicação da cobertura especial adequada para a prevenção ou tratamento da lesão ${ }^{22,23}$.

O conhecimento sobre a cobertura adequada a ser utilizada depende do conhecimento sobre composição, mecanismo de ação, indicação, advertências, modo de usar e intervalo de troca de cada opção. Saber avaliar e identificar as necessidades da ferida complementa a decisão sobre qual objetivo deve ser alcançado para otimização daquele processo cicatricial.

Foi possível observar que mesmo os enfermeiros com capacitação/aperfeiçoamento enfrentam dificuldades para indicar a cobertura devido ao tipo de indicação de cada produto. Os dados chamam a atenção, pois o atendimento na APS tem um manual com padronização para os tratamentos das feridas. As limitações do estudo referem-se ao número de participantes e à dificuldade geográfica para acesso a todas as unidades da APS do município pesquisado. Após a finalização da pesquisa, os resultados foram apresentados para os gestores da SMS.

\section{CONCLUSÃO}

Os resultados do estudo permitiram avaliar que, mesmo os enfermeiros tendo recebido curso de capacitação/ aperfeiçoamento sobre tratamento de feridas ofertado pela SMS, a porcentagem de indicação de cada cobertura especial foi inferior a $50 \%$ em quase todas as questões pesquisadas, o que denota conhecimento insuficiente sobre o tema.

Quando realizada a comparação entre o grupo de enfermeiros que tem capacitação/aperfeiçoamento em feridas e o que não tem, não há diferença estatisticamente significante. A cobertura com maior porcentagem de acerto foi o hidrogel, e o com indicação inadequada foi o hidrocoloide.

É possível inferir que, em decorrência de conhecimento insuficiente para indicação das coberturas especiais, a atuação do enfermeiro especialista nessa área contribuiria de forma significativa para o paciente, no que se refere ao tratamento da ferida e ao serviço, quanto ao desperdício de material.

Espera-se que este estudo possa servir como fulcro para futuras investigações na área, bem como para uma possível revisão na reordenação dos serviços de APS que realizam curativos especiais.

\section{CONTRIBUIÇÃO DOS AUTORES}

Conceitualização, Caveião C e Hey AP; Metodologia, Caveião C; Hey AP e Sales WB; Investigação, Tavares ELP e Souza E; Redação - Primeira versão, Caveião C; Tavares ELP e Souza E; Redação - Revisão \& Edição, Caveião C; Hey AP; Sales WB e Silva MMBG; Supervisão, Caveião C.

\section{REFERÊNCIAS}

1. Garnelo L, Lucas ACS, Parente RCP, Rocha ESC, Gonçalves MJF. Organização do cuidado às condições crônicas por equipes de saúde da família na Amazônia. Saúde Debate. 2014;38(Espec):158-72. $\quad$ https://doi.org/10.5935/01031104.20145012

2. Ministério da Saúde. Secretaria de Atenção à Saúde. Departamento de Atenção Básica (BR). Normas e Manuais Técnicos. Cadernos de Atenção Primária, n. 29. Brasília: MS; 2010 [citado 11 dez 2017]. Disponível em: http:// bvsms.saude.gov.br/bvs/publicacoes/caderno atencao primaria 29 rastreamento.pdf.

3. Azevedo IC, Costa RKS, Holanda CSM, Salvetti MG, Torres GV. Conhecimento de enfermeiros da estratégia saúde da família sobre avaliação e tratamento de feridas oncológicas. Rev Bras Cancerol. 2014;60(2):119-27.

4. Cavalcante BLL, Lima UTS. Relato de experiência de uma estudante de enfermagem em um consultório especializado em tratamento de feridas J Nurs Health. 2012;1(2):91-103. 
5. Conselho Federal de Enfermagem. Resolução COFEN No 567 de 29 de janeiro de 2018 [Internet]. Brasília, DF; 2018. [citado 07 nov 2018]. Disponível em: http://www.cofen.gov. br/resolucao-cofen-no-567-2018_60340.html.

6. Smaniotto PHS, Ferreira MC, Isaac C, Galli R. Systematization of dressings for clinical treatment of wounds. Rev Bras Cir Plást. 2012;27(4):623-66. https://doi.org/10.1590/S198351752012000400026.

7. National Pressure Ulcer Advisory Panel. NPUAP Pressure Injury Stages [Internet]. Washington: NPUAP; 2016 [citado 11 dez 2017]. Disponivel em:_http://www.npuap.org/resources/educationaland-clinical-resources/npuap-pressure-injury-stages/.

8. Leite AP, Oliveira BGRB, Soares MF, Barrocas DLR. Uso e efetividade da papaína no processo de cicatrização de feridas: uma revisão sistemática. Rev Gaúcha Enferm. 2012;33(3):198-207. https://doi.org/10.1590/S198314472012000300026 .

9. Cunha MB, Sousa LRM, Castro JMSS, Melo GL, Sousa LRG, Carvalho ML. Avaliação do conhecimento da equipe de enfermagem de um hospital público sobre a prática de curativo. Rev Interd. 2015;8(1):83-90.

10. Conselho Nacional de Saúde. Resolução No 466 de 12 de dezembro de 2012. [Internet]. Brasília, DF; 2012. [citado 11 dez 2017]. Disponível em: http://conselho.saude.gov.br/ resolucoes/2012/reso466.pdf.

11. Souza LL, Araújo DB, Silva DS, Bêrredo VC. Representações de gênero na prática de enfermagem na perspectiva de estudantes. Ciência \& Cognição. 2014;19(2):218-32.

12. Silva KL, Sena RR, Tavares TS, Martins ACS. Oferta de cursos de graduação de enfermagem no estado de Minas Gerais. Texto contexto - enferm. 2011;20(Espec):124-130. https:// doi.org/10.1590/S0104-07072011000500016.

13. Conselho Nacional de Educação. Câmara de Educação Superior. Resolução CNE/CES N 03 de 7 de novembro DE 2001 [Internet]. Brasília, DF; 2001. [citado 11 dez 2017]. Disponível em: http://portal.mec.gov.br/cne/arquivos/pdf/ CES03.pdf.

14. Salomé GM. Avaliando lesão: práticas e conhecimento dos enfermeiros que prestam assistência ao indivíduo com ferida. Saúde Coletiva. 2009;6(35):280-287.
15. Erdmann AL, Peiter CC, Lanzoni GMM. Brazilian research groups in nursing: comparison of 2006 and 2016 profiles. Rev Gaúcha Enferm. 2017;38(2):e69051. https://doi. org/10.1590/1983-1447.2017.02.69051.

16. Prado AR, Barreto VPM, Tonini T, Silva AS, Machado WCA. O saber do enfermeiro na indicação de coberturas no cuidado ao cliente com feridas. ESTIMA, Braz J Enterostomal Ther. 2016;14(4):175-82. https://doi.org/10.5327/Z18063144201600040004.

17. Silva DRA, Bezerra SMG, Costa JP, Luz MHBA, Lopes VCA, Nogueira LT. Pressure ulcer dressings in critical patients: a cost analysis. Rev esc enferm USP. 2017;51:e03231. https:// doi.org/10.1590/s1980-220x2016014803231.

18. Melo EM, Fernandes VS. Avaliação do conhecimento do enfermeiro acerca das coberturas de última geração. ESTIMA, Braz J Enterostomal Ther. 2011;9(4).

19. Silva ACO, Rodrigues Filho ESF, Sousa GRS, Silva JFS, Silva $\mathrm{AL}$, Araujo CMS. As principais coberturas utilizadas pelo enfermeiro. Revista Uningá. 2017;53(2):117-23.

20. Canadian Association of Wound Care. Foundations of best practice for skin and wound management. Best practice recommendations for the prevention and management of wounds [Internet]. Toronto; 2016. [citado $11 \mathrm{dez} 2017$ ]. Disponível em: https://www.woundscanada.ca/docman/ public/health-care-professional/bpr-workshop/165-wc-bprprevention-and-management-of-wounds/file.

21. Anjos ET, Teixeira LA, Rodrigues MA, Paula MAB, Vasconcellos ACLP. O que os enfermeiros de saúde coletiva sabem sobre o tratamento das úlceras venosas. ESTIMA, Braz J Enterostomal Ther. 2007;5(4).

22. Teixeira AKS, Menezes LCG, Oliveira RM. Serviço de estomaterapia na perspectiva dos gerentes de enfermagem em hospital público de referência. ESTIMA, Braz J Enterostomal Ther. 2016;14(1):3-12. https://doi. org/10.5327/Z1806-3144201600010002.

23. Chayamiti EMPC, Yano TK, Mabtum A, Carmo DHP, Garcia MLB, Viliod MCL, et al. Dificuldades para o uso de inovações: assistência às pessoas com feridas crônicas nas unidades de saúde de Ribeirão Preto. ESTIMA, Braz J Enterostomal Ther. 2007;5(3). 